\title{
Impact of Channel Knowledge on Cognitive Radio System Capacity
}

\author{
Pawel A. Dmochowski*, Himal A. Suraweera ${ }^{\dagger}$, Peter J. Smith ${ }^{\ddagger}$, Mansoor Shafi ${ }^{\S}$ \\ * School of Engineering and Computer Science, Victoria University of Wellington, Wellington, New Zealand \\ $\dagger$ Department of Electrical and Computer Engineering, National University of Singapore, Singapore \\ $\ddagger$ Department of Electrical and Computer Engineering, University of Canterbury, Christchurch, New Zealand \\ $\S$ Telecom New Zealand, Wellington, New Zealand \\ Email:pdmochowski@ieee.org, elesaha@nus.edu.sg,p.smith@elec.canterbury.ac.nz, mansoor.shafi@telecom.co.nz
}

\begin{abstract}
We examine the impact of channel knowledge on the secondary user (SU) in a cognitive radio system. Under a minimum signal-to-interference-and-noise ratio (SINR) constraint for the primary user (PU) receiver, we determine the SU capacity under four channel knowledge scenarios. We derive analytical expressions for the capacity cumulative distribution functions which are verified by means of simulations. We show that the lack of exact knowledge of the PU-PU channel gain by the SU-Tx either prohibits SU transmission or necessitates high interference level at the PU. We also show that the lack of exact knowledge of the SU-Tx to PU-Rx link has little or no impact on SU capacity.
\end{abstract}

\section{INTRODUCTION}

The cognitive radio concept, introduced in [1], refers to a smart radio which can sense the external electromagnetic environment and adapt its transmission parameters according to the current state of the environment. Secondary (or Cognitive) users (SUs) can be designed to access parts of the primary user (PU) spectrum for their information transmission, provided they cause minimal interference to the PUs in that band [2].

A large number of papers have appeared on various aspects of cognitive radio systems, including fundamental information theoretic capacity limits (see, for example, [3]-[10]). The cognitive radios can protect the PU transmissions by regulating the SU transmitter to PU receiver interference power to be below a well defined threshold level. The limits on this received interference level at the PU receiver can be imposed with an average/peak constraint [4]. Another method of protecting the $\mathrm{PU}$ transmission is to consider a minimum value for its signal-to-interference noise ratio (SINR) beyond which further degradation is not accepted. Note that if the PU signal has a signal-to-noise ratio (SNR) below this level then the SU cannot transmit at all since the minimum SINR is unobtainable. Clearly with the SINR constraint, depending on the fading level of the PU-Tx to PU-Rx link, the conservatism inherent in the constant interference threshold constraint can be relaxed to some extent. Hence, with an SINR constraint, the tolerable PU interference is no longer a constant and this can be to the benefit of the SU-Tx when the PU link is strong. The price of this relaxation is that some information about the PU-Tx to PU-Rx link must be available to the SU-Tx.

The motivation for our work arises from the fact that complete channel knowledge acquisition of all links in the network is impractical. Generally in wireless systems, various

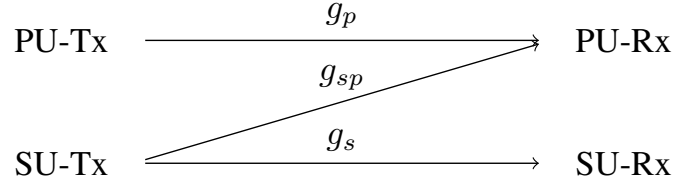

Fig. 1. System Model

levels of channel knowledge (either in the form of instantaneous or statistical channel information) on radio links are collected for system optimization. Moreover, due to estimation errors, the acquired channel information is never perfect. Therefore, an important question is the impact of the nature of channel knowledge availability on the cognitive radio capacity. Previously, [6] derived SU capacity under a peakpower constraint and asymmetric channel conditions, while [7] examined the impact of instantaneous channel estimation errors on the SU capacity. Note that [7] only considered imperfect channel state information (CSI) for the SU-PU link. In this paper we consider the effect of statistical CSI rather than instantaneous channel estimation errors. Furthermore, the investigation considers the effect of statistical CSI on all the channels for the primary and secondary links under both SINR and peak power constraints.

\section{SYSTEM MODEL}

As shown in Fig. 1, we consider a cognitive radio system where a SU link consisting of a SU-Tx and a SU-Rx transmits simultaneously over the same narrow band with a PU link consisting of a PU-Tx and a PU-Rx. Independent point-to-point flat Rayleigh fading channels are assumed for all links in the network. Let $g_{p}=\left|h_{p}\right|^{2}, g_{s}=\left|h_{s}\right|^{2}$ and $g_{s p}=\left|h_{s p}\right|^{2}$ denote the instantaneous channel gains of the PU-Tx to PU-Rx, SUTx to SU-Rx and SU-Tx to PU-Rx links, respectively. Furthermore, we denote the exponentially distributed probability density functions (pdfs) of the random variables (RVs) $g_{p}, g_{s}$ and $g_{s p}$ by $f_{g_{p}}(x), f_{g_{s}}(x)$, and $f_{g_{s p}}(x)$, respectively. These distributions are governed by their corresponding parameters $\Omega_{p}=\mathbb{E}\left(g_{p}\right), \Omega_{s}=\mathbb{E}\left(g_{s}\right)$ and $\Omega_{s p}=\mathbb{E}\left(g_{s p}\right)$, where $\mathbb{E}(\cdot)$ denotes the expectation operator. In this paper, depending on the type of channel knowledge available at the SU-Tx, we identify four different scenarios of interest. In all cases, it is 
assumed that the SU-Tx has exact knowledge of $g_{s}{ }^{1}$

Scenario 1: The PU-Tx to PU-Rx channel $g_{p}$ and SU-Tx to the PU-Rx channel $g_{s p}$ are perfectly known.

Scenario 2: The PU-Tx to PU-Rx channel $g_{p}$ is perfectly known while only the mean $\Omega_{s p}$ of the channel between the SU-Tx and the PU-Rx is known.

Scenario 3: The mean $\Omega_{p}$ and the exact channel gain $g_{s p}$ are known.

Scenario 4: Only the means $\Omega_{p}$ and $\Omega_{s p}$ are known.

In all cases, where possible, we impose a constraint, $\gamma_{T}$, on the SINR at the PU receiver, denoted by $\gamma_{p}$. Hence,

$$
\gamma_{p}=\frac{P_{p} g_{p}}{P_{s} g_{s p}+\sigma_{p}^{2}}, \text { and } \gamma_{p} \geq \gamma_{T}
$$

where $\gamma_{T}$ is a pre-defined SINR threshold, $P_{p}$ and $P_{s}$ are the primary and secondary transmit powers, respectively, and $\sigma_{p}^{2}$ is the additive white Gaussian noise (AWGN) variance at the PURx. Note that in the event that the PU-Rx SNR $P_{p} g_{p} / \sigma_{p}^{2}<\gamma_{T}$, the SINR constraint in (1) cannot be satisfied, and thus the SU transmit power is zero. We also impose a maximum SU transmit power constraint, denoted by $P_{m}$, which arises in practice, for example, due to power amplifier nonlinearities. Thus, in Scenarios 1 and 2, where the SU-Tx knows $g_{p}$, the $\mathrm{SU}$ transmit power is given by

$$
P_{t}=\left\{\begin{array}{ll}
0 & \frac{P_{p} g_{p}}{\gamma_{T}}<\sigma_{p}^{2} \\
\min \left(P_{s}, P_{m}\right) & \text { otherwise }
\end{array},\right.
$$

where $P_{s}$ is obtained from (1) by solving $\gamma_{T}=\gamma_{p}$. As will be shown in Section III, the $P_{t}=0$ condition for Scenarios 3 and 4 will depend on the choice of system parameters and thus (2) will be modified accordingly.

Furthermore, we note that the constraints described above can only be guaranteed if the SU-Tx has perfect knowledge of the links $g_{p}$ and $g_{s p}$, that is under Scenario 1. In analysing Scenarios 2-4, we use probabilistic contraints. Hence, we require the SINR constraint to hold with an acceptably high probability, $1-\alpha$, where $\alpha$ is small.

The signal-to-noise ratio at the SU-Rx is $\frac{P_{t} g_{s}}{\sigma_{s}^{2}}$, where $\sigma_{s}^{2}$ is the AWGN variance at the SU-Rx. In our investigation of the SU capacity $C$, we first consider the RV $\gamma=P_{t} g_{s}$, with the pdf and cumulative distribution function (cdf) denoted by $f_{\gamma}(x)$ and $F_{\gamma}(x)$, respectively. The SU capacity is thus given by

$$
C=\log _{2}\left(1+\frac{\gamma}{\sigma_{s}^{2}}\right)
$$

which allows us to obtain the cdf of $C$ from $F_{\gamma}(x)$ by

$$
F_{C}(y)=F_{\gamma}\left(\sigma_{s}^{2}\left(2^{y}-1\right)\right) .
$$

\footnotetext{
${ }^{1}$ The impact of the interference caused by the PU-Tx to SU-Rx link on the SU capacity is not taken into account due to space constraints. Analysis involving this factor is, however, considered in the forthcoming full journal version of this work.
}

Furthermore, the mean capacity, $\bar{C}$, can be derived using $f_{\gamma}(x)$ by

$$
\bar{C}=\mathbb{E}(C)=\int_{0}^{\infty} \log _{2}\left(1+\frac{x}{\sigma_{s}^{2}}\right) f_{\gamma}(x) d x .
$$

In what follows we derive analytical expressions for $F_{\gamma}(x)$ and $f_{\gamma}(x)$ which, using (4) and (5), allow us to compute the capacity cdf and mean capacity for each scenario.

\section{SU CAPACITY}

\section{A. Scenario 1}

Assuming that the SU has full knowledge of $g_{p}, g_{s}$ and $g_{s p}$, $P_{s}$ can be obtained directly from (1), giving

$$
P_{s}=\frac{\frac{P_{p} g_{p}}{\gamma_{T}}-\sigma_{p}^{2}}{g_{s p}}
$$

We note that while we ignore the $P_{t}=0$ case in (2), the following derivation is valid since $\operatorname{Pr}(\gamma>0)=0$ for $P_{t} \leq 0$. In finding $F_{\gamma}(x)$ we solve for the complementary cdf of $\gamma$

$$
\begin{aligned}
\operatorname{Pr}(\gamma>x) & =\operatorname{Pr}\left(g_{s} \min \left(P_{m}, P_{s}\right)>x\right) \\
& =\operatorname{Pr}\left(P_{m} g_{s}>x,\left(\frac{P_{p} g_{p}}{\gamma_{T}}-\sigma_{p}^{2}\right) \frac{g_{s}}{g_{s p}}>x\right) \\
& =\operatorname{Pr}\left(g_{s}>\frac{x}{P_{m}},\left(\frac{P_{p} g_{p}}{\gamma_{T}}-\sigma_{p}^{2}\right) g_{s}>x g_{s p}\right)
\end{aligned}
$$

where $\operatorname{Pr}(\cdot)$ denotes probability. Noting that $g_{p}$ is an exponentially distributed RV, we can rewrite (7) as

$\operatorname{Pr}(\gamma>x)=\int_{0}^{\infty} \int_{\frac{x}{P_{m}}}^{\infty} e^{-\frac{\gamma_{T}}{P_{p} \Omega_{p}}\left(\frac{x v}{u}+\sigma_{p}^{2}\right)} f_{g_{s}}(u) f_{g_{s p}}(v) d u d v$

Substituting for $f_{g_{s}}(u)$ and $f_{g_{s p}}(v)$, and changing the order of integration one obtains

$$
\begin{aligned}
\operatorname{Pr} & \gamma>x) \\
& =\frac{e^{-\frac{\gamma_{T} \sigma_{p}^{2}}{P_{p} \Omega_{p}}}}{\Omega_{s p} \Omega_{s}} \int_{\frac{x}{P_{m}}}^{\infty} e^{-\frac{u}{\Omega_{s}}} \int_{0}^{\infty} e^{-\left(\frac{\gamma_{T} x}{P_{p} \Omega_{p} u}+\frac{1}{\Omega_{s p}}\right) v} d v d u \\
& =\frac{e^{-\frac{\gamma_{T} \sigma_{p}^{2}}{P_{p} \Omega_{p}}}}{\Omega_{s p} \Omega_{s}} \int_{\frac{x}{P_{m}}}^{\infty} \frac{e^{-\frac{u}{\Omega_{s}}}}{\frac{\gamma_{T} x}{P_{p} \Omega_{p} u}+\frac{1}{\Omega_{s p}}} d u .
\end{aligned}
$$

After simplifying (9), the cdf $F_{\gamma}(x)=1-\operatorname{Pr}(\gamma>x)$ can be shown to be [11, Equation (3.351.2)]

$$
\begin{aligned}
F_{\gamma}(x) & =1-e^{-\frac{\gamma_{T} \sigma_{p}^{2}}{P_{p} \Omega_{p}}}\left[e^{-\frac{x}{P_{m} \Omega_{s}}}-\frac{\Omega_{s p} \gamma_{T} x}{P_{p} \Omega_{p} \Omega_{s}} e^{\frac{\Omega_{s p} \gamma_{T} x}{P_{p} \Omega_{p} \Omega_{s}}}\right. \\
& \left.\times \Gamma\left(0, \frac{\Omega_{s p} \gamma_{T} x}{P_{p} \Omega_{p} \Omega_{s}}+\frac{x}{P_{m} \Omega_{s}}\right)\right],
\end{aligned}
$$

where $\Gamma(\cdot, \cdot)$ is the upper incomplete gamma function. Finally, the capacity cdf is obtained by substituting (10) into (4). 
Furthermore, we can obtain the pdf $f_{\gamma}(x)$ by differentiating (10) with respect to $x$, which after some manipulation gives

$$
\begin{aligned}
f_{\gamma}(x) & =e^{-\frac{\gamma_{T} \sigma_{p}^{2}}{P_{p} \Omega_{p}}}\left[\left(\frac{1}{P_{m} \Omega_{s}}-\frac{\Omega_{s p} \gamma_{T}}{P_{p} \Omega_{p} \Omega_{s}}\right) e^{-\frac{x}{P_{m} \Omega_{s}}}\right. \\
& +e^{\frac{\Omega_{s p} \gamma_{T} x}{P_{p} \Omega_{p} \Omega_{s}}}\left(\frac{\left(\Omega_{s p} \gamma_{T}\right)^{2} x}{\left(P_{p} \Omega_{p} \Omega_{s}\right)^{2}}+\frac{\Omega_{s p} \gamma_{T}}{P_{p} \Omega_{p} \Omega_{s}}\right) \\
& \left.\times \Gamma\left(0, \frac{\Omega_{s p} \gamma_{T} x}{P_{p} \Omega_{p} \Omega_{s}}+\frac{x}{P_{m} \Omega_{s}}\right)\right] .
\end{aligned}
$$

Equation (11) can be used in (5) to give a single numerical integral for the mean capacity $\bar{C}$.

\section{B. Scenario 2}

Note that in Scenarios 2-4, exact channel knowledge is unavailable. As a result, the SU cannot guarantee that (1) is satisfied since the value of $\gamma_{p}$ is uncertain. Hence, we constrain the SU to satisfy (1) with an acceptably high probability, $1-\alpha$, where $\alpha$ is usually small.

Specifically, in Scenario 2, where the SU knows only the mean, $\Omega_{s p}$, of $g_{s p}$, we consider the probability of satisfying the SINR constraint with a probability of $1-\alpha$, that is

$$
\operatorname{Pr}\left(\frac{P_{p} g_{p}}{P_{s} g_{s p}+\sigma_{p}^{2}} \geq \gamma_{T} \mid g_{p}, \Omega_{s p}\right)=1-\alpha,
$$

which can be rewritten as

$$
\operatorname{Pr}\left(g_{s p} \leq \frac{P_{p} g_{p}-\gamma_{T} \sigma_{p}^{2}}{P_{s} \gamma_{T}} \mid g_{p}, \Omega_{s p}\right)=1-\alpha .
$$

From (13) we can derive the expression for the transmit power $P_{s}$, that is

$$
P_{s}=-\frac{P_{p} g_{p}-\gamma_{T} \sigma_{p}^{2}}{\gamma_{T} \Omega_{s p} \ln (\alpha)} .
$$

From (14) the complementary cdf of $\gamma$ is derived by noting that

$$
\begin{aligned}
& \operatorname{Pr}(\gamma>x)=\operatorname{Pr}\left(P_{m} g_{s}>x, P_{s} g_{s}>x\right) \\
& \quad=\operatorname{Pr}\left(g_{s}>\frac{x}{P_{m}} \mid P_{m}<P_{s}\right)+\operatorname{Pr}\left(g_{s}>\frac{x}{P_{s}} \mid P_{m}>P_{s}\right),
\end{aligned}
$$

which in turn can be shown to be

$$
\begin{aligned}
\operatorname{Pr}(\gamma>x) & =\int_{\psi_{0}}^{\psi} \operatorname{Pr}\left(g_{s}>\frac{x}{P_{s}}\right) f_{g_{p}}(y) d y \\
& +\int_{\psi}^{\infty} \operatorname{Pr}\left(g_{s}>\frac{x}{P_{m}}\right) f_{g_{p}}(y) d y,
\end{aligned}
$$

where $\psi_{0}=\frac{\gamma_{T} \sigma_{p}^{2}}{P_{p}}$ and $\psi=\frac{\gamma_{T}\left(\sigma_{p}^{2}-P_{m} \Omega_{s p} \ln (\alpha)\right)}{P_{p}}$. The lower integration limit in the first term of (16) takes into account the $P_{t}=0$ condition in (2). After some manipulation, we can simplify (16) to obtain $F_{\gamma}(x)=1-\operatorname{Pr}(\gamma>x)$ as,

$$
\begin{aligned}
F_{\gamma}(x) & =1-\int_{\psi_{0}}^{\psi} \operatorname{Pr}\left(g_{s}>\frac{x}{P_{m}}\right) f_{g_{p}}(y) d y \\
& -\int_{\psi}^{\infty} \operatorname{Pr}\left(g_{s}>-\frac{x \gamma_{T} \Omega_{s p} \ln (\alpha)}{P_{p} y-\gamma_{T} \sigma_{p}^{2}}\right) f_{g_{p}}(y) d y,
\end{aligned}
$$

which results in

$$
\begin{aligned}
F_{\gamma}(x) & =1-\exp \left\{-\frac{x}{P_{m} \Omega_{s}}-\frac{\psi}{\Omega_{p}}\right\} \\
& -\frac{1}{\Omega_{p}} \int_{\psi_{0}}^{\psi} e^{-\frac{x \gamma_{T} \Omega_{s p} \ln (\alpha)}{\left(\gamma_{T} \sigma_{p}^{2}-P_{p} y\right) \Omega_{s}}} e^{-\frac{y}{\Omega_{p}}} d y .
\end{aligned}
$$

To the best of our knowledge, there exists no closed form solution to the integral in (18), and in Section IV it is evaluated numerically.

\section{Scenario 3}

In Scenario 3, where the SU has exact knowledge of $g_{s p}$ and knows only the mean $\Omega_{p}$, we once again satisfy the SINR constraint with a probability of $1-\alpha$. Hence,

$$
\operatorname{Pr}\left(\frac{P_{p} g_{p}}{P_{s} g_{s p}+\sigma_{p}^{2}} \geq \gamma_{T} \mid \Omega_{p}, g_{s p}\right)=1-\alpha,
$$

which gives

$$
\operatorname{Pr}\left(g_{p} \geq \frac{\gamma_{T}\left(P_{s} g_{s p}+\sigma_{p}^{2}\right)}{P_{p}} \mid \Omega_{p}, g_{s p}\right)=1-\alpha .
$$

Following the same approach as for Scenario 2 in Section III-B, noting that $g_{p}$ is an exponential RV, one can show that

$$
P_{s}=-\left(\frac{\ln (1-\alpha) P_{p} \Omega_{p}}{\gamma_{T}}+\sigma_{p}^{2}\right) \frac{1}{g_{s p}} .
$$

Defining for notational convenience

$$
Q=-\left(\frac{\ln (1-\alpha) P_{p} \Omega_{p}}{\gamma_{T}}+\sigma_{p}^{2}\right),
$$

we have a transmit power for Scenario 3 given by

$$
P_{t}=\left\{\begin{array}{ll}
0 & Q<0 \\
\min \left(P_{m}, \frac{Q}{g_{s p}}\right) & Q>0
\end{array} .\right.
$$

Using (22) and (23) we note that the SU transmission will only occur for $Q>0$, which is equivalent to

$$
\alpha>1-e^{-\frac{\gamma_{T}}{P_{p} \Omega_{p} / \sigma_{p}^{2}}} .
$$

In such a case, solving for the cdf of $\gamma$, we write

$\operatorname{Pr}(\gamma>x)$

$$
=\operatorname{Pr}\left(g_{s}>\frac{x}{P_{m}} \mid P_{m}<\frac{Q}{g_{s p}}\right)+\operatorname{Pr}\left(g_{s}>\frac{x}{P_{s}} \mid P_{m}>\frac{Q}{g_{s p}}\right)
$$

giving

$$
\begin{aligned}
\operatorname{Pr}(\gamma>x) & =\int_{0}^{Q / P_{m}} \operatorname{Pr}\left(g_{s}>\frac{x}{P_{m}}\right) f_{g_{s p}}(y) d y \\
& +\int_{Q / P_{m}}^{\infty} \operatorname{Pr}\left(g_{s}>\frac{x y}{Q}\right) f_{g_{s p}}(y) d y .
\end{aligned}
$$

Upon simplifying (26), we obtain

$$
\begin{aligned}
F_{\gamma}(x) & =1-e^{-\frac{x}{\Omega_{s p} P_{m}}}\left(1-e^{-\frac{Q}{\Omega_{s} P_{m}}}\right) \\
& -\frac{1}{1+\frac{\Omega_{s} x}{\Omega_{s p} Q}} e^{-\frac{Q}{\Omega_{s p} P_{m}}-\frac{x}{\Omega_{s} P_{m}}} .
\end{aligned}
$$


Here, once again we can obtain the pdf

$$
\begin{aligned}
f_{\gamma}(x) & =\frac{1-e^{-\frac{Q}{\Omega_{s p} P_{m}}}}{\Omega_{s} P_{m}} e^{-\frac{x}{\Omega_{s} P_{m}}} \\
& +\frac{1}{\Omega_{s} P_{m}} \frac{e^{-\frac{Q}{\Omega_{s p} P_{m}}-\frac{x}{\Omega_{s} P_{m}}}}{1+\frac{\Omega_{s p}}{\Omega_{s} Q} x}+\frac{\Omega_{s p}}{\Omega_{s} Q} \frac{e^{-\frac{Q}{\Omega_{s p} P_{m}}-\frac{x}{\Omega_{s} P_{m}}}}{\left(1+\frac{\Omega_{s p}}{\Omega_{s} Q}\right)^{2}} .
\end{aligned}
$$

Using (28) and (5), the mean SU capacity can be shown to be

$$
\begin{aligned}
\bar{C} & =\frac{1-e^{-\frac{Q}{\Omega_{s p} P_{m}}}}{\ln (2)} \Gamma\left(0, \frac{\sigma_{s}^{2}}{\Omega_{s} P_{m}}\right) e^{\frac{\sigma_{s}^{2}}{\Omega_{s} P_{m}}} \\
& -\frac{1}{\ln (2)\left(1-\frac{\Omega_{s p} \sigma_{s}^{2}}{\Omega_{s} Q}\right)} \Gamma\left(0, \frac{Q}{\Omega_{s p} P_{m}}\right) \\
& +\frac{e^{-\frac{Q}{\Omega_{s p} P_{m}}}}{\ln (2)\left(1-\frac{\Omega_{s p} \sigma_{s}^{2}}{\Omega_{s} Q}\right)} \Gamma\left(0, \frac{\sigma_{s}^{2}}{\Omega_{s} P_{m}}\right) e^{\frac{\sigma_{s}^{2}}{\Omega_{s} P_{m}}} .
\end{aligned}
$$

\section{Scenario 4}

Consider the final scenario, where the SU-Tx has knowledge of only the mean values of $g_{p}$ and $g_{s p}$. Here we have

$$
\operatorname{Pr}\left(\frac{P_{p} g_{p}}{P_{s} g_{s p}+\sigma_{p}^{2}} \geq \gamma_{T} \mid \Omega_{p}, \Omega_{s p}\right)=1-\alpha .
$$

Using conditioning (30) can be given as

$$
\mathbb{E}\left[\operatorname{Pr}\left(P_{p} g_{p} \geq \gamma_{T}\left(P_{s} g_{s p}+\sigma_{p}^{2}\right) \mid g_{s p}\right)\right]=1-\alpha,
$$

which after some manipulation gives the transmit power $P_{s}$,

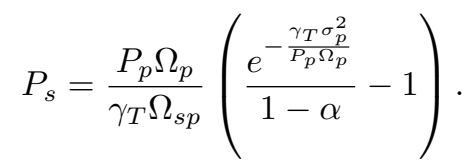

Here, $P_{s}$ and thus $P_{t}$, are deterministic, depending simply on the system parameters. The latter is given by

$$
P_{t}=\left\{\begin{array}{ll}
0 & P_{s}<0 \\
P_{s} & 0<P_{s}<P_{m} \\
P_{m} & P_{s}>P_{m}
\end{array} .\right.
$$

Similarly to Scenario 3, from (32) and (33) we conclude that SU transmission occurs when (24) is satisfied.

The cdf of $\gamma$ is given by

$$
F_{\gamma}(x)=1-e^{-\frac{x}{P_{t} \Omega_{s}}},
$$

and, from (4), the capacity cdf is

$$
F_{C}(y)=1-e^{-\frac{\sigma_{p}^{2}\left(2^{y}-1\right)}{P_{t} \Omega_{s}}}
$$

The pdf of $C$ can be trivially obtained by differentiating (35). Furthermore, we can evaluate the mean SU capacity using (5),

$$
\begin{aligned}
\bar{C} & =\frac{1}{\ln (2) P_{t} \Omega_{s}} \int_{0}^{\infty} \ln \left(1+\frac{x}{\sigma_{s}^{2}}\right) e^{-\frac{x}{P_{t} \Omega_{s}}} d x \\
& =\frac{1}{\ln (2)} e^{\frac{\sigma_{s}^{2}}{P_{t} \Omega_{s}}} E_{1}\left(\frac{\sigma_{s}^{2}}{P_{t} \Omega_{s}}\right)
\end{aligned}
$$

where $E_{1}(x)=\int_{x}^{\infty} e^{-t} / t d t$ denotes the exponential integral function of order one.

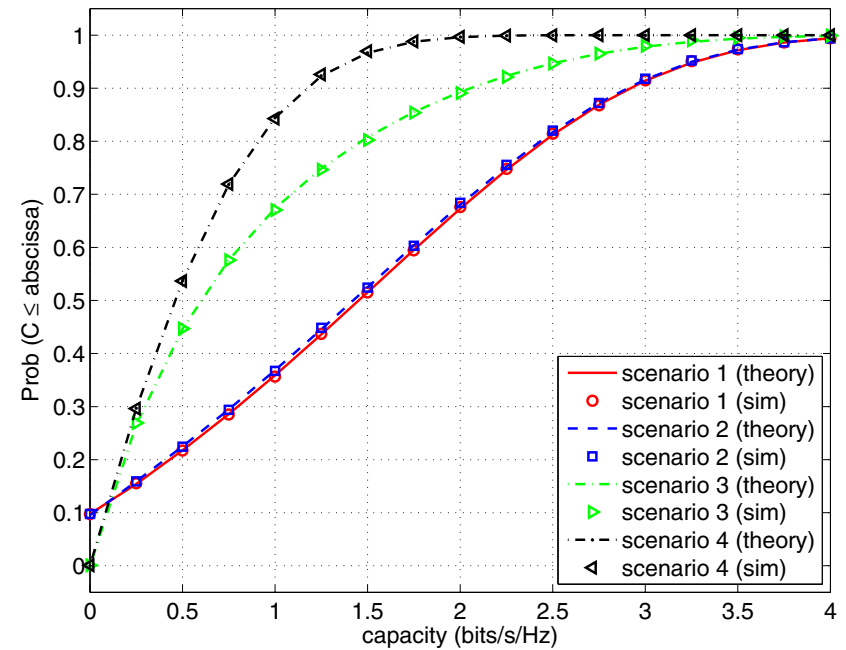

Fig. 2. SU capacity cdf for Scenarios $1-4\left(c_{1}=c_{2}=0.1\right)$.

\section{Simulation Results And Discussion}

We now present simulation results used to validate the analytical expressions derived in Section III, and to compare capacity values achievable under each scenario. In all simulations we have set $P_{p} / \sigma_{p}^{2}=P_{m} / \sigma_{s}^{2}=0 \mathrm{~dB}$ and $\Omega_{p} / \sigma_{p}^{2}=\Omega_{s} / \sigma_{s}^{2}=5 \mathrm{~dB}$, where we assume $\sigma_{p}^{2}=\sigma_{s}^{2}$. In Scenarios 2-4 we set $\alpha=0.1$. The remaining system variables were parameterized using

$$
c_{1}=\frac{\Omega_{s p}}{\Omega_{s}},
$$

that is, the ratio of interference to desired channel strength, and

$$
c_{2}=\frac{\gamma_{T}}{P_{p} \Omega_{p} / \sigma_{p}^{2}},
$$

which is the ratio of the minimum target SINR to the actual $\mathrm{SNR}$ at the PU-Rx. Hence, increasing $c_{2}$ corresponds to reducing the allowable interference, with the case of $c_{2}=1$ corresponding to zero allowable interference. The parameter values used in the simulations are indicated in the relevant figures.

Figures 2 and 3 show the SU capacity cdf for all scenarios, with lines corresponding to analytical expressions using (10), (18), (27) and (35), and symbols indicating simulatated results. From these results we observe that Scenarios 1 and 2 result in similar performance, even in the case of $c_{1}=0.9$, that is where the SU interference is very prominent, thus making it difficult to satisfy the PU SINR constraint (1). Furthermore, lack of knowledge of the PU-PU (that is, knowing only the mean $\Omega_{p}$ ) greatly reduces the achievable capacity of the SU. From the point of view of the SU-PU collaboration this is a positive result, since $g_{p}$ is likely to be known at the PU-Rx. Hence, the PU already has the most useful information and does not need to estimate $g_{s p}$, which might make the price of co-existence too high from the PU perspective.

In comparing all four scenarios in Figs. 2 and 3, we used $c_{2}=0.1$, which is very generous towards the SU. 


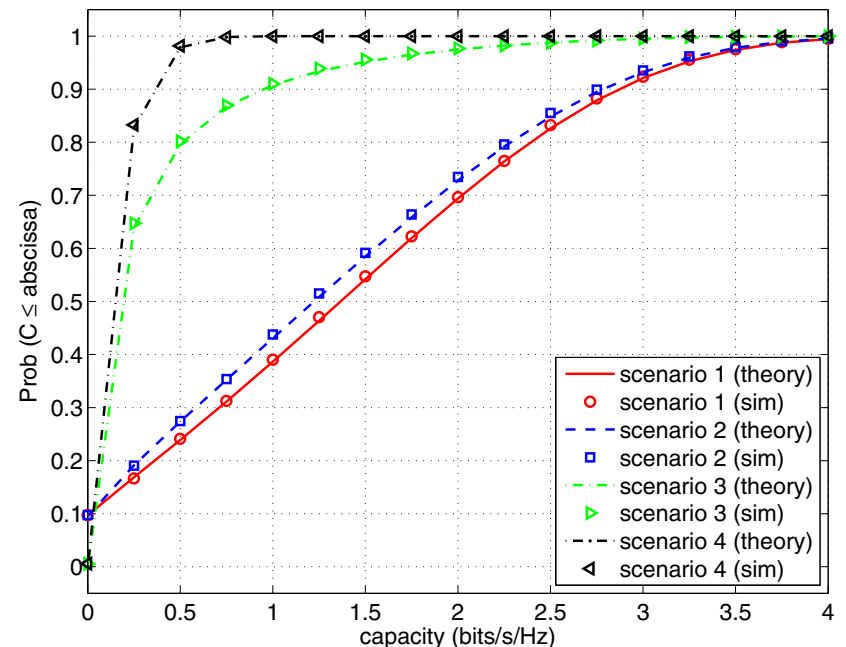

Fig. 3. SU capacity cdf for Scenarios $1-4\left(c_{1}=0.9, c_{2}=0.1\right)$.

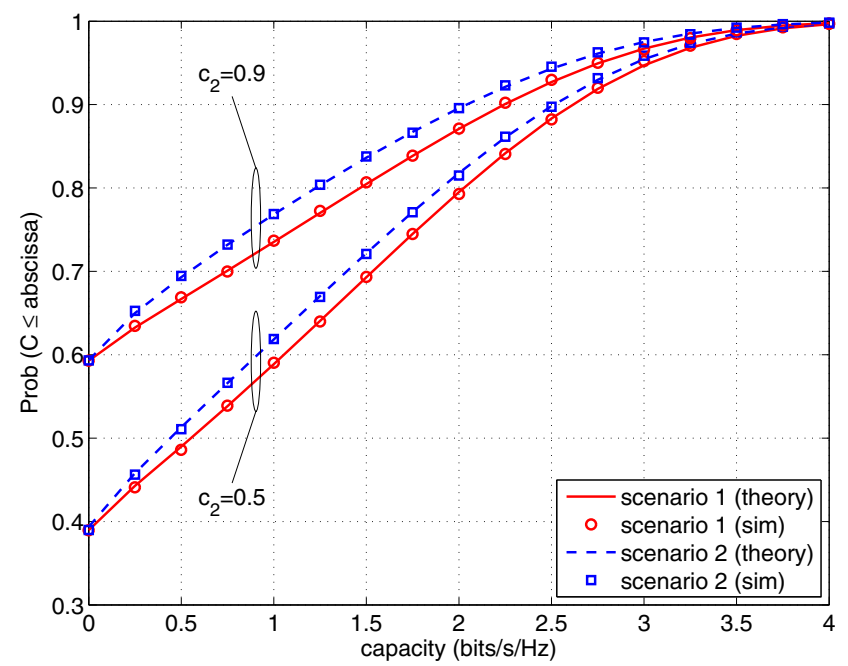

Fig. 4. SU capacity cdf for Scenarios 1 and $2\left(c_{1}=0.1 ; c_{2}=0.5\right.$, $\left.c_{2}=0.9\right)$

Recalling (24), and noting that using (38) it can be written as $\alpha>1-e^{-c_{2}}$, we see that SU transmission in Scenarios 3 and 4 occurs only for large values of $\alpha$ or for small values of $c_{2}$. That is, without the knowledge of $g_{p}$, the SU can only operate if the $\mathrm{PU}$ is willing to accept large amounts of interference. Figures 4 presents the capacity results for Scenarios 1 and 2 with more realistic values of $c_{2}=0.5$ and $c_{2}=0.9$, where (24) prevents SU transmission under Scenarios 3 and 4. Finally, Fig. 5 shows the probability $\operatorname{Pr}(C \leq 1)$, where capacity is measured in $\mathrm{bps} / \mathrm{Hz}$, as a function of $c_{1}$. As expected, for a constant $c_{2}$, the performance under Scenario 2 diverges from the baseline Scenario 1 with increasing $c_{1}$, that is as the amount of interference to the PU increases.

\section{CONCLUSIONS}

In this paper, we have examined the effects of channel knowledge availability on the capacity of the SU. Considering four scenarios, we derived analytical expressions for the SU

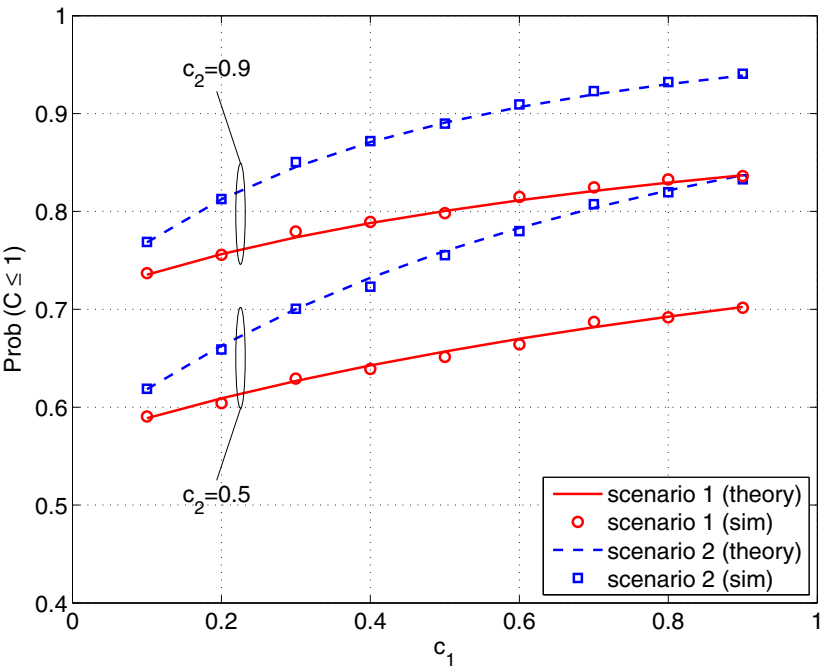

Fig. 5. SU capacity cdf for Scenarios 1 and 2 for varying $c_{1}\left(c_{2}=0.5\right.$, $\left.c_{2}=0.9\right)$.

capacity cdf under the constraint of a minimum SINR at the PU-Rx, which were verified by means of Monte Carlo simulations. The results demonstrate the importance of the PU-PU CSI, which was shown to be much greater than that of the SU-Tx to PU-Rx link. More importantly, we show that for reasonable system parameters SU transmission does not occur unless the PU-PU CSI is known.

\section{REFERENCES}

[1] J. Mitola III, "Cognitive radio: An integrated agent architecture for software defined radio," Ph.D. dissertation, KTH Royal Institute of Technology, Sweden, May 2000.

[2] T. A. Weiss and F. K. Jondral, "Spectrum pooling: An innovative stratergy for the enhancement of spectrum efficiency," IEEE Commun. Mag., vol. 42, pp. 8-14, March 2004.

[3] S. A. Jafar and S. Srinivasa, "Capacity limits of cognitive radio with distributed and dynamic spectral activity," IEEE J. Sel. Areas Commun., vol. 25, pp. 529-537, April 2007.

[4] A. Ghasemi and E. S. Sousa, "Fundamental limits of spectrum-sharing in fading environments," IEEE Trans. Wireless Commun., vol. 6, pp. 649-658, February 2007.

[5] L. Musavian and S. Aissa, "Fundamental capacity limits of spectrumsharing channels with imperfect feedback," in Proc. IEEE GLOBECOM 2007, November 2007, pp. 1385-1389.

[6] H. A. Suraweera, J. Gao, P. J. Smith, M. Shafi, and M. Faulkner, "Channel capacity limits of cognitive radio in asymmetric fading environments," in Proc. IEEE ICC 2008, May 2008, pp. 4048-4053.

[7] H. A. Suraweera, P. J. Smith, and M. Shafi, "Capacity limits and performance analysis of cognitive radio with imperfect channel knowledge," IEEE Trans. Veh. Technol., accepted, January 2010.

[8] R. Zhang, "Optimal power control over fading cognitive radio channels by exploiting primary user CSI," in Proc. IEEE GLOBECOM 2008, November 2008, pp. 1-5.

[9] X. Kang, Y.-C. Liang, A. Nallanathan, H. K. Garg, and R. Zhang, "Optimal power allocation for fading channels in cognitive radio networks: Ergodic capacity and outage capacity," IEEE Trans. Wireless Commun., vol. 8, pp. 940-950, February 2009.

[10] C.-X. Wang, X. Hong, H.-H. Chen, and J. Thompson, "On capacity of cognitive radio networks with average interference power constraints," IEEE Trans. Wireless Commun., vol. 8, pp. 1620-1625, April 2009.

[11] I. S. Gradshteyn and I. M. Ryzhik, Table of Integrals, Series and Products, 7th ed. San Diego, CA: Academic Press, 2007. 